\title{
Patienten nachlässig bei oraler Antikoagulation
}

\author{
Um die Therapieadhärenz gegenüber oralen Antikoagulanzien ist es in der Praxis schlecht bestellt. \\ Auch die Einführung der DOAK hat daran nicht viel geändert, wie eine Studie aus den USA zeigt
}

\begin{abstract}
_ In einer retrospektiven Kohortenanalyse wurden für den Zeitraum November 2010 bis Dezember 2014 die Daten von 64.661 Patienten mit Vorhofflimmern analysiert, die unter einer Therapie mit Warfarin, Dabigatran, Rivaroxaban oder Apixaban standen. Die mediane Beobachtungszeit betrug 1,1 Jahre. Von den Patienten, die ein direktes orales Antikoagulans (DOAK) nahmen, erreichten nur 47,5\% eine Abdeckung von $\geq 80 \%$ der Tage. Bei den Patienten unter Warfarin waren es sogar nur 40,2\%.

Patienten mit einem $\mathrm{CHA}_{2} \mathrm{DS}_{2}$-VAScScore von $\geq 4$ hatten ein fast doppelt so hohes Risiko für einen Schlaganfall, wenn sie ihre Medikation über mehr als einen Monat nicht eingenommen hatten,
\end{abstract}

als Patienten, die weniger als eine Woche nicht unter Antikoagulation standen. Bei einem $\mathrm{CHA}_{2} \mathrm{DS}_{2}$-VASc-Score von 2-3 machte sich dieses erhöhte Risiko erst bemerkbar, wenn die Zeit ohne Antikoagulation $\geq 6$ Monate betrug (Hazard Ratio: 2,73).

Bei Patienten mit $\mathrm{CHA}_{2} \mathrm{DS}_{2}$-VAScScore von 0 oder 1 bestand keine Korrelation zwischen Schlaganfällen und der Zeitdauer ohne Antikoagulation. Hingegen machte sich in dieser Gruppe eine Zeit ohne Antikoagulation von $\geq 3$ Monaten durch eine signifikante Verringerung der Blutungsereignisse bemerkbar. Dieses letzte Ergebnis spricht für die Validität des Modells im Sinne einer internen Kontrolle der Daten.
- Yao X et al. Effect of adherence to oral anticoagulation on risk of stroke and major bleeding among patients with atrial fibrillation. J Am Heart Assoc. 2016;5:e003074

\section{KOMMENTAR}

Die Untersuchung hat zwei wesentliche Aspekte. Zum einen dürfen wir uns bezüglich der Adhärenz bei der Antikoagulation keinen Illusionen hingeben. Die schönen Daten aus den frühen Studien werden wir in der Praxis wahrscheinlich niemals erreichen können. Gerade unter dem Aspekt der ungenügenden Adhärenz sollte man sich die Indikation zur Antikoagulation bei Patienten mit einem $C H A_{2} D S_{2}$-VASC-Score von 0 oder 1 besonders sorgfältig überlegen, da hier die Vorteile der Antikoagulation die Risiken möglicherweise nicht überwiegen.

Prof. Dr. med. H. S. FüeßI

\section{Malignes Geschehen am Augenlid}
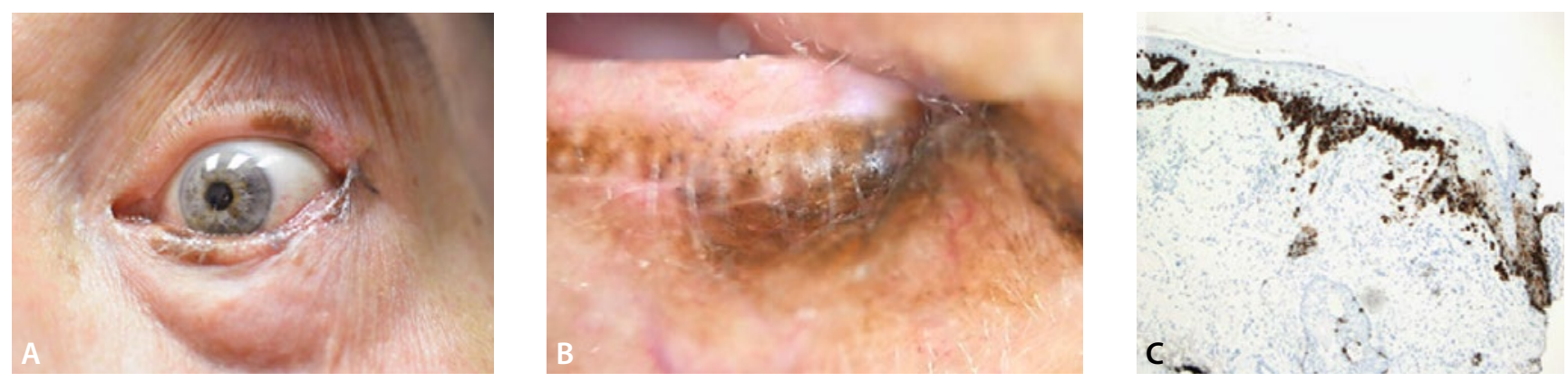

A, B: Dunkle Hautverfärbungen an den Lidern des linken Auges. C: Histologischer Befund (Melan-A-Färbung).

Ein 87-jähriger Mann wurde zur Therapie eines Gerstenkorns am linken Oberlid überwiesen. Trotz konservativer Therapie war das Gerstenkorn nicht zurückgegangen. Dem Hausarzt fiel auf, dass sich die Haut dunkel verfärbt hatte (Abb. A und B). Der Patient berichtete über Juckreiz, hatte aber keine Schmerzen. Er hatte über viele Jahrzehnte als Strandwächter gearbeitet und war dadurch sehr stark der Sonne ausgesetzt. Eine Biopsie aus dem Bereich ergab ein invasives malignes Melanom mit einer Tumordicke nach Breslow von 0,35 mm ohne Ulzeration und ohne Mitogenese (Abb. C). Der Patient wurde für die Melanombehandlung an eine Spezialklinik überwiesen. Allerdings verweigerte er jede Therapie und war auch nicht mehr zu weiteren Untersuchungen bereit.

Primäre Melanome des Augenlids sind selten und machen weniger als $1 \%$ aller kutanen Melanome aus. Die Lentigo maligna ist der häu- figste histologische Subtyp des Melanoms am Augenlid. Das untere Augenlid ist häufiger betroffen als das obere, da es noch mehr dem Sonnenlicht ausgesetzt ist. Wenn sich Hautbefunde bei älteren Menschen über längere Zeit nicht bessern, sollte man immer an ein malignes $\mathrm{Ge}$ schehen denken.

Prof. Dr. med. H. S. FüeßI

- MarchalikRJ, Venna S (rjb254@georgetown.edu). Eyelid melanoma. N Engl J Med. 2016;375:75 